\title{
Amadeus
}

International Multidisciplinary Journal ISSN 2525-8281

V.2, N. 4. Mar./2018 - ISSN 2525-8281

\section{BLADDER CANCER AND DEBATE ON TRIMODAL THERAPY (TMT) FOR BLADDER PRESERVATION}

\begin{abstract}
Antonio
Marlos Duarte de Melo, ${ }^{1}$ Jose Angelo Araújo Sampaio, ${ }^{1}$ Ana Beatriz Callou Sampaio Neves, ${ }^{1}$ Isabela Callou Sampaio Neves ${ }^{2}$, Lucas Leimig Telles Parente ${ }^{l}$, Maria Valeria Leimig Telles ${ }^{l}$, Mikael Vieira da Silva ${ }^{3}$

Abstract: Bladder Cancer is one of the most common types of malignant neoplasms that affects both men and women. The surgery considered standard for the treatment of invasive tumor is Radical Cystectomy, which causes undesirable morbidity in the postoperative period on affected patients. Radical Transurethral Resection (TRU), associated with Chemotherapy and Radiotherapy, together form the Trimodal Therapy (TTM), which can demonstrate satisfactory results in the approach of the patient with invasive tumor with lower morbidity and well-timed survival rate, in a short time.

Keywords: Bladder Cancer; Radical Cystectomy; Transurethral Resection; Trimodal Therapy.
\end{abstract}

\section{Introduction}

Malignant bladder neoplasm is one of the leading causes of cancer in the world. ${ }^{1,2,3}$ The major challenge of bladder cancer is the patient's quality of life, which may become fragile due to the removal of the bladder. ${ }^{4}$ Today, Bladder cancer represents the fourth most common neoplasm and occupies the eighth place as the cause of death from malignant disease, being more common in males, smokers, the elderly and, with nosocomial manipulation in the urinary tract, presence of urinary calculi, exposure to aniline dyes and infection with Schistosoma haematobium. ${ }^{1,5}$.

\footnotetext{
${ }^{1}$ Faculty of Medicine Estacio of Juazeiro do Norte (FMJ), Juazeiro do Norte, Ceara, Brazil. Contact: marlos_duarte@outlook.com

${ }^{2}$ City Hall of Juazeiro do Norte, Juazeiro do Norte, Ceara, Brazil.

${ }^{3}$ Pronutrir Cariri Oncology, Juazeiro do Norte, Ceara, Brazil.
} 
The initial clinical course of the disease may manifest itself with hematuria in $85 \%$ of patients, in addition to dysuria and flank pain, but these are non-specific symptoms, which may also occur in benign diseases such as Urinary Tract Infection, Nephrolithiasis and Benign Prostatic Hyperplasia. ${ }^{1}$

Conventional cystoscopy is still a standard exam to detect the bladder tumor, but it may not be advantageous to detect Carcinoma in situ (CIS). In addition to other reasons, it requires histopathological confirmation, evidencing in about $90 \%$ of them the existence of Transitional Cell Carcinoma, which is the most common malignant tumor in the bladder. Besides that, it is important to perform imaging tests such as CT scan and Magnetic Nuclear Resonance for suitable staging. ${ }^{1,2}$ Fortunately, 70 to $80 \%$ of the patients at diagnosis are in a noninvasive form, which is not present in the muscular layer. ${ }^{1}$

\section{Treatment}

The gold standard treatment for Invasive Bladder Cancer is still the Radical Cystectomy (RC), but using Neoadjuvant Chemotherapy before the surgical procedure. ${ }^{1-7}$ The Transurethral Bladder Tumor Resection (TUBTR) gives prognosis, is diagnostic and often therapeutic in some circumstances, but after an initial TUR, many patients (34\% to $76 \%$ ) will have residual disease present due to non-resection of the tumor in its entirety, increasing the risk of recurrence and progression. ${ }^{1}$

In non-invasive tumor, the minimum goal of bladder cancer treatment is to eliminate the tumor, prevent disease recurrence and a possible progression to invasive muscle disease. In this way, it aims the decrease the specific mortality of the disease by treating these patients with TUBTR, the administration of a single chemotherapeutic in the immediate postoperative period and, after treatment, follow-up with Surveillance Cystoscopy due to frequency of recurrence. ${ }^{1}$

For invasive muscle tumor, $\mathrm{RC}$ is still the standard gold treatment, performing the radical surgical procedure as soon as possible, since the delay of this approach implies a lower survival due to the progression of the disease with the presence of micrometastases. ${ }^{1-7}$

Hospitals that perform a high volume of radical cystectomies have lower mortality rates and complications than low volume centers. ${ }^{1}$ In men, the standard $\mathrm{RC}$ is the removal of the bladder, prostate, vesicles Seminal and distal ureters, where as in women, RC removes 
bladder, urethra, vagina, uterus and ureters. The so-called Laparoscopic Radical Cystectomy has been recently employed, giving potential advantages such as less blood loss, smaller incisions, less bowel exposure to the external environment, but requires long-term research for proper acceptance as an option equivalent to the open path. ${ }^{1,5}$ Like patients with noninvasive tumors, those affected by invasive malignancy should have a follow-up because of the risk of tumor recurrence, which may give symptoms of hematuria and flank pain. ${ }^{1,5}$

\section{Debate}

Preservation of the bladder is perhaps the most interesting current topic for advancing the approach to the patient with invasive bladder tumor, since most patients are elderly and this can provide a choice of treatment and a better quality of life due to lower morbidity. ${ }^{2,3,8}$ Organ preservation therapies have been developed as an alternative to radical treatments for cancer diseases located in organs. ${ }^{7}$

The debate is whether this therapy can achieve the same survival rates that are obtained with radical cystectomy; however, because of the lack of long-term randomized studies, this comparison is still unknown. ${ }^{1,4,7}$ When the bladder is to be preserved, the treatment of invasive tumor is performed with Trimodal Therapy (TMT), which determines a surgical approach with radical TUR, radiotherapy and chemotherapy, selecting well the patients who should undergo this therapeutic line for a correct comparative study with the other therapies. $3,4,6,7,8$

Patients with tumor-related hydronephrosis, flat lesions such as CIS, locally advanced disease (T4), presence of urethral obstruction and lymph node involvement are poor candidates for preservation of the bladder and are generally excluded from TMT. ${ }^{3,4}$ Tumors eligible for preservation of the bladder are those with low volume T2 disease without hydronephrosis, CIS; Single tumors less than three centimeters and of usual histology; And patients who can't or don't want to undergo RC. ${ }^{4}$

In addition, even in patients who are not ideal candidates for bladder preservation therapy and who are not surgical candidates, they should still be offered TMT because cure can still be achieved. ${ }^{7}$ In cases of tumor recurrence to TMT, the patient should undergo Rescue Cystectomy. For metastatic tumors, the survival rate is somber, only 12-15 months. ${ }^{1}$ 
Accordingly, a growing body of research suggests that TMT - with salvage cystectomy reserved for tumor recurrence or Unresponsive - leads to acceptable results and may be considered a reasonable treatment option in well-selected patients. ${ }^{4}$ The similarity in survival between patients who undergo RC and TMT is probably due to the rapid use of rescue cystectomy in cases of recurrence in the bladder preservation series. ${ }^{5,6,8}$

\section{Final Considerations}

Therefore, new studies are needed to elucidate this comparison between radical cystectomy and TMT, aiming to improve the quality of life of the patient affected by bladder cancer, because the majority of cases represent localized tumors and should not mean the immediate withdrawal of the bladder. These studies should aim to indicate the true advantages of TMT over radical cystectomy, which is a procedure that increases patient morbidity. Thus, the importance of a debate about the advantages of TMT for the preservation of the bladder in the person with malignant neoplasia in this organ is a fact, also allowing the reduction of relapses and not progression to the invasive disease.

\section{How to cite this article (APA format):}

Melo, Antonio M. D. de.; Sampaio, José A. A.; Neves, Ana B. C. S.; Neves, Isabela C. S.; Parente, Lucas L. T.; Telles, Maria V. L.; Silva, Mikael V. da. (2018). Bladder cancer and debate on trimodal therapy (tmt) for bladder preservation. Am Int Mul J, 2(4), 52-56.

Received: 04/16/2018.

Accepted: 04/18/2018.

\section{References}

1 Jacobs, B., Lee, C. and Montie, J. (2010). Bladder Cancer in 2010: How Far have We Come?.CA: A Cancer Journal for Clinicians, 60(4), pp.244-272.

2 James, N., Hussain, S., Hall, E., Jenkins, P., Tremlett, J., Rawlings, C., Crundwell, M., 
Sizer, B.,Sreenivasan, T.,Hendron, C., Lewis, R., Waters, R. and Huddart, R. (2012). Radiotherapy with or without Chemotherapy in Muscle-Invasive Bladder Cancer. New England Journal of Medicine, 366(16), pp.1477-1488.

3 Premo, C., Apolo, A., Agarwal, P. and Citrin, D. (2015). Trimodality Therapy in Bladder Cancer. Urologic Clinics of North America, 42(2), pp.169-180.

4 Ploussard, G., Daneshmand, S., Efstathiou, J., Herr, H., James, N., Rödel, C., Shariat, S., Shipley, W., Sternberg, C., Thalmann, G. and Kassouf, W. (2014). Critical Analysis of Bladder Sparing with Trimodal Therapy in Muscle-invasive Bladder Cancer: A Systematic Review. European Urology, 66(1), pp.120-137.

5 Gakis, G., Efstathiou, J., Lerner, S., Cookson, M., Keegan, K., Guru, K., Shipley, W., Heidenreich, A., Schoenberg, M., Sagaloswky, A., Soloway, M. and Stenzl, A. (2013). ICUD-EAU International Consultation on Bladder Cancer 2012: Radical Cystectomy and Bladder Preservation for Muscle-Invasive Urothelial Carcinoma of the Bladder. European Urology, 63(1), pp.45-57.

6 Kulkarni, G., Hermanns, T., Wei, Y., Bhindi, B., Satkunasivam, R., Athanasopoulos, P., Bostrom, P., Kuk, C., Li, K., Templeton, A., Sridhar, S., van der Kwast, T., Chung, P., Bristow, R., Milosevic, M., Warde, P., Fleshner, N., Jewett, M., Bashir, S. and Zlotta, A. (2017). Propensity Score Analysis of Radical Cystectomy Versus Bladder-Sparing Trimodal Therapy in the Setting of a Multidisciplinary Bladder Cancer Clinic. Journal of Clinical Oncology, pp.JCO.2016.69.232.

7 Mitin, T. (2017). Radical Cystectomy is the best choice for most patients with muscleinvasive bladder cancer? Opinion: No. International brazjurol, 43(2), pp.188-191.

8 Solsona, E., Iborra, I., Collado, A., Rubio-Briones, J., Casanova, J. And Calatrava, A. (2010). Feasibility of Radical Transurethral Resection as Monotherapy for Selected Patients With Muscle Invasive Bladder Cancer. The Journal of Urology, 184(2), pp.475-481. 